\title{
Origin of the near-Earth asteroid Phaethon and the Geminids meteor shower
}

\author{
J. de León ${ }^{1}$, H. Campins ${ }^{2}$, K. Tsiganis ${ }^{3}$, A. Morbidelli ${ }^{4}$, and J. Licandro ${ }^{5,6}$ \\ 1 Instituto de Astrofísica de Andalucía-CSIC, Camino Bajo de Huétor 50, 18008 Granada, Spain \\ e-mail: jleon@iaa.es \\ 2 University of Central Florida, PO Box 162385, Orlando, FL 32816.2385, USA \\ e-mail: campins@physics.ucf.edu \\ 3 Department of Physics, Aristotle University of Thessaloniki, 54124 Thessaloniki, Greece \\ 4 Departement Casiopée: Universite de Nice - Sophia Antipolis, Observatoire de la Côte d'Azur, CNRS 4, 06304 Nice, France \\ 5 Instituto de Astrofísica de Canarias (IAC), C/Vía Láctea s/n, 38205 La Laguna, Spain \\ 6 Department of Astrophysics, University of La Laguna, 38205 La Laguna, Tenerife, Spain
}

Received 5 November 2009 / Accepted 26 January 2010

\section{ABSTRACT}

\begin{abstract}
Aims. In this paper we establish a compositional and dynamical connection between two B-type objects: main belt asteroid (2) Pallas and near-Earth asteroid (3200) Phaethon. The final purpose is to help understand the origin of this very interesting object.

Methods. We first compare visible and near-infrared spectra of asteroids Phaethon and Pallas. We then compare the reflectance spectra of Phaethon with all the available visible spectra of B-type asteroids belonging to the Pallas family. One last spectral comparison is then performed to search for any correspondence between Phaethon and any B-type asteroid in the main belt. Numerical simulations are also carried out to explore the dynamical connection between the orbital neighborhoods of Pallas and Phaethon.

Results. Main differences between Phaethon and Pallas lie in the visible wavelength part of their reflectance spectra. We have also found that the nine asteroids belonging to the Pallas family have visible spectra that are different from that of Pallas and strikingly similar to that of Phaethon. Spectral comparison excludes any other B-type asteroid in the main belt as a possible parent body of Phaethon. Numerical simulations establish a dynamical pathway that connects Phaethon with Pallas and its family members.

Conclusions. The spectral similarities between Phaethon and Pallas family members, together with their established dynamical connection, supports Pallas as the most likely parent body of Phaethon and therefore, the associated Geminids meteor stream. We suggest that differences in asteroid sizes are the most likely explanation for the differences in the visible reflectance spectra between Phaethon and Pallas.
\end{abstract}

Key words. minor planets, asteroids: general - techniques: spectroscopic - methods: numerical

\section{Introduction}

Asteroid (3200) Phaethon is a remarkable near-Earth asteroid (NEA). It was the first asteroid associated with a meteor shower, namely the Geminid stream (Whipple 1983), and together with 2005 UD and 2001 YB5 is one of the 3 NEAs with associated meteor showers. Phaethon's unusual orbit has a high inclination $\left(i=22.18^{\circ}\right)$ and a very low perihelion distance $(0.14 \mathrm{AU})$. Its reflectance spectrum suggests a connection with primitive meteorites, best fitting with $\mathrm{CI} / \mathrm{CM}$ carbonaceous chondrites (Licandro et al. 2007; Emery et al. 2008), aqueously altered and rich in hydrated silicates. Following the most recent taxonomy of DeMeo et al. (2009), it is classified as a B-type object. Recent studies suggest a connection with the population of main-belt comets (Hsieh \& Jewitt 2006), classifying Phaethon as an activated asteroid.

Asteroid Pallas is one of the largest objects in the main asteroid belt, with a diameter of $\sim 550 \mathrm{~km}$. This asteroid has also unusual orbital parameters for such a large body: it has a highly inclined orbit $\left(i \sim 34^{\circ}\right)$ and a high orbital eccentricity $(e=0.23)$. Classified as a primitive, B-type object, it has been widely related to carbonaceous chondrites (Chapman \& Salisbury 1973; Johnson \& Fanale 1973; Johnson \& Matson 1975) and with evidence of hydration (Lebofsky 1980; Rivkin et al. 2002). Lemaitre \& Morbidelli (1994) were the first to note the existence of a possible collisional family around Pallas.

In this paper we show a possible connection between near-Earth asteroid Phaethon and main belt asteroid Pallas. For Pallas to be considered as a likely source for Phaethon, it must fulfill the following requirements. First, Phaethon must be spectroscopically similar to Pallas and its family members, which would suggest similar surface composition. Second, a dynamical mechanism must exist, such that Pallas fragments of Phaethon's size (a diameter of $\sim 5 \mathrm{~km}$ ) can develop planet-crossing orbits and become highly-inclined NEAs. In the following sections we show that both requirements are met.

\section{Data compilation and family members comparison}

To explore the compositional connection between Phaethon and Pallas, we have compiled the relevant spectra from several databases. The visible spectra $(0.45-0.92 \mu \mathrm{m})$ of Pallas, Pallas family members and Phaethon, were taken from the SMASS survey (Bus \& Binzel 2001a,b; Binzel et al. 2001, 2004). The 

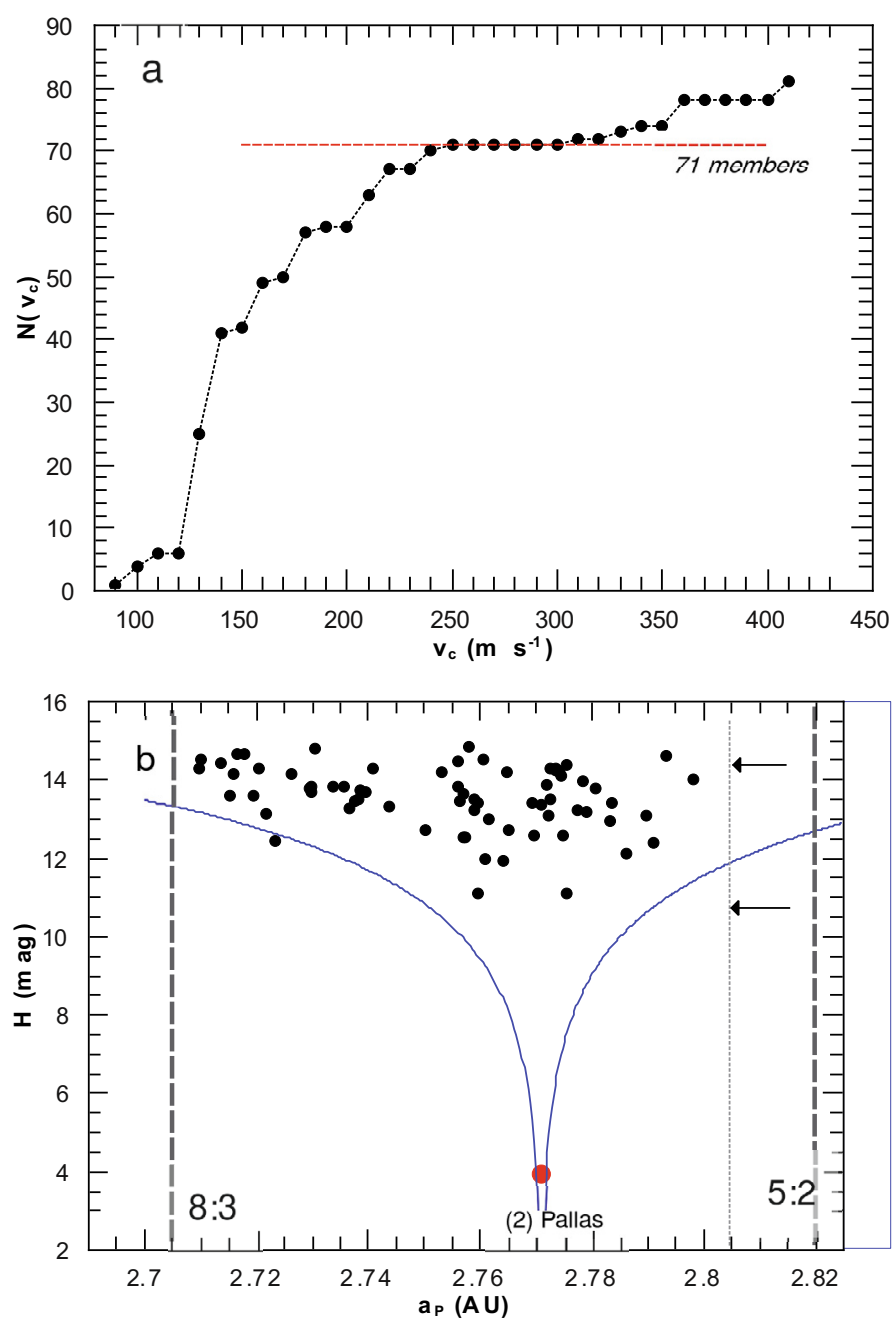

Fig. 1. Determination of asteroids that belong to the Pallas family. a) Number of asteroids against the velocity cut-off $\left(V_{\mathrm{c}}\right)$ used in the HCM to define the family. For $V_{\mathrm{c}}=300 \mathrm{~m} \mathrm{~s}^{-1}$, there are 71 possible Pallas family members (red dashed line). b) Distribution of the Pallas family members in proper semi-major axis and absolute visual magnitude, $\left(a_{\mathrm{p}}, H\right)$. The vertical dashed lines show the 8:3 and the 5:2 MMRs. The arrows indicate the range in $H$ in which the Pallas family is contained.

near-infrared spectra $(0.8-2.5 \mu \mathrm{m})$ of both Pallas and Phaethon were taken from the MIT-UH-IRTF survey (Binzel et al. 2008). For the sake of comparison we have also used visible and nearinfrared spectra of Phaethon from the NEOSS database (de León et al. 2010), previously published by Licandro et al. (2007).

To determine which asteroids belong to the Pallas family, we apply the hierarchical clustering method (HCM, Zappalà et al. 1995) on the latest catalog of numerically computed proper elements of all numbered asteroids (provided by the AstDyS service, http: //hamilton. unipi.it/astdys). The number of asteroids linked to Pallas changes with the considered value of velocity cut-off, $V_{c}$, which is a measure of the distance between two bodies in proper elements space. We can identify a family of 40-80 members, for a velocity cut-off in the range $V_{\mathrm{c}}=130-400 \mathrm{~m} \mathrm{~s}^{-1}$. The number of asteroids linked to the family varies with $V_{\mathrm{c}}$, reaching a plateau for $V_{\mathrm{c}}=240-360 \mathrm{~m} \mathrm{~s}^{-1}$ (see Fig. 1a). These high values of $V_{c}$ suggest that this family represents a cratering event (also indicated by observations, e.g. Schmidt et al. 2009), rather than a catastrophic disruption, given that Pallas probably contains $>99 \%$ of the family's mass. In that
Table 1. Proper orbital elements and physical parameters for Pallas and 9 Pallas family members classified as B-types.

\begin{tabular}{lcccccc}
\hline \hline $\begin{array}{l}\text { Ast. } \\
\text { Num. }\end{array}$ & $\begin{array}{c}a \\
(\mathrm{AU})\end{array}$ & $e$ & $\begin{array}{c}i \\
\left({ }^{\circ}\right)\end{array}$ & $H$ & $p_{\mathrm{v}}$ & $\begin{array}{c}D \\
(\mathrm{~km})\end{array}$ \\
\hline 2 & 2.771 & 0.281 & 33.198 & 3.93 & $0.16 \pm 0.01$ & 546.02 \\
531 & 2.786 & 0.254 & 33.055 & 11.80 & $0.15 \pm 0.01$ & 15.19 \\
2382 & 2.759 & 0.274 & 32.949 & 11.12 & $0.15^{a}$ & 26.00 \\
3579 & 2.733 & 0.283 & 33.089 & 13.83 & $0.15^{a}$ & 5.88 \\
5222 & 2.775 & 0.277 & 32.688 & 11.11 & $0.15 \pm 0.01$ & 21.92 \\
5234 & 2.760 & 0.271 & 33.123 & 11.98 & $0.15^{a}$ & 13.78 \\
5330 & 2.764 & 0.261 & 31.848 & 11.94 & $0.22 \pm 0.04$ & 12.00 \\
\hline 3200 & 1.271 & 0.889 & 22.184 & 14.51 & $0.11 \pm 0.01$ & 5.10 \\
\hline $3581^{b}$ & 2.771 & 0.409 & 28.810 & 12.10 & $0.15^{a}$ & 13.04 \\
$5690^{b}$ & 2.804 & 0.379 & 28.956 & 12.50 & $0.15^{a}$ & 20.85 \\
$6500^{b}$ & 2.756 & 0.417 & 29.282 & 12.50 & $0.15^{a}$ & 10.85 \\
\hline
\end{tabular}

Notes. ${ }^{(a)}$ For those objects that do not have IRAS albedo information, we have used the nominal value 0.15 . $^{(b)}$ Although classified as Marscrossers, we show in the main text that these 3 asteroids belong in fact to the Pallas family. As in the case of Phaethon, osculating orbital elements have been used.

case, multi-kilometer sized fragments could have been ejected from Pallas with relative velocities comparable to the escape velocity of Pallas ( $320 \mathrm{~m} \mathrm{~s}^{-1}$; see e.g. Asphaug 1997, for the Vesta case).

For a velocity cut-off of $V_{\mathrm{c}}=300 \mathrm{~m} \mathrm{~s}^{-1}$, we find 71 possible Pallas family members. Visible spectra are available only for six of these asteroids, all of which are classified as B-types. The proper orbital elements and some physical parameters of Pallas and its family members are shown in Table 1 . We include the semi-major axis $(a)$, eccentricity $(e)$ and inclination (i) of their orbits, as well as their absolute visual magnitude $(H)$ and their visual geometric albedo $\left(p_{\mathrm{v}}\right)$, the later taken from IRAS database (Tedesco 1992). Diameter is estimated from $H$ and $p_{\mathrm{v}}$ following the expression of Fowler \& Chillemi (1992), $2 \log (D)=6.247-0.4 H-\log \left(p_{\mathrm{v}}\right)$. The differences between the albedo of Phaethon and Pallas family members are within the error bars ${ }^{1}$. For asteroid Phaethon, osculating orbital elements have been used, taken from JPL Horizons online database (http://ssd.jpl.nasa.gov). Its albedo value has been taken from Harris (1998), where they use the NEATM model, more appropriate for a small and fast rotating asteroid than the STM model used within IRAS database.

The distribution of the Pallas family members in proper semi-major axis and absolute visual magnitude $\left(a_{\mathrm{p}}, H\right)$ appears to be "V"-shaped (see Fig. 1b), a phenomenon known to be associated both with size-dependent ejection velocities field as well as with a drift in $a_{\mathrm{p}}$ due to the Yarkovsky effect. Note that the Pallas family is contained within and abruptly terminates at the location of two mean motion resonances (MMRs) with Jupiter (the 8:3 MMR, at $a \sim 2.71 \mathrm{AU}$, and the 5:2 MMR, at $a \sim 2.82 \mathrm{AU})$. The minimum value of absolute magnitude $(H)$ of asteroids near the inner truncation distance (the 8:3 MMR with Jupiter) is $H \sim 14.2$, which translates to a diameter $D=4.95 \mathrm{~km}$ (using a mean albedo of $p_{\mathrm{v}}=0.15$ ), i.e. these bodies are the size of Phaethon. Thus, some Phaethon-sized Pallas family fragments could have been able to reach these resonances. Those that

\footnotetext{
${ }^{1}$ Uncertainties in the diameter estimated with thermal modelling usually exceed the formal errors, and are typically $10-15 \%$ (e.g. Delbo et al. 2003), which translated to albedo means uncertainties of 20-30\%. For $p_{\mathrm{v}}=0.15$ that means errors of $0.03-0.05$, larger than the nominal errors included in the IRAS database.
} 
J. de León et al.: Connection between Phaethon and Pallas

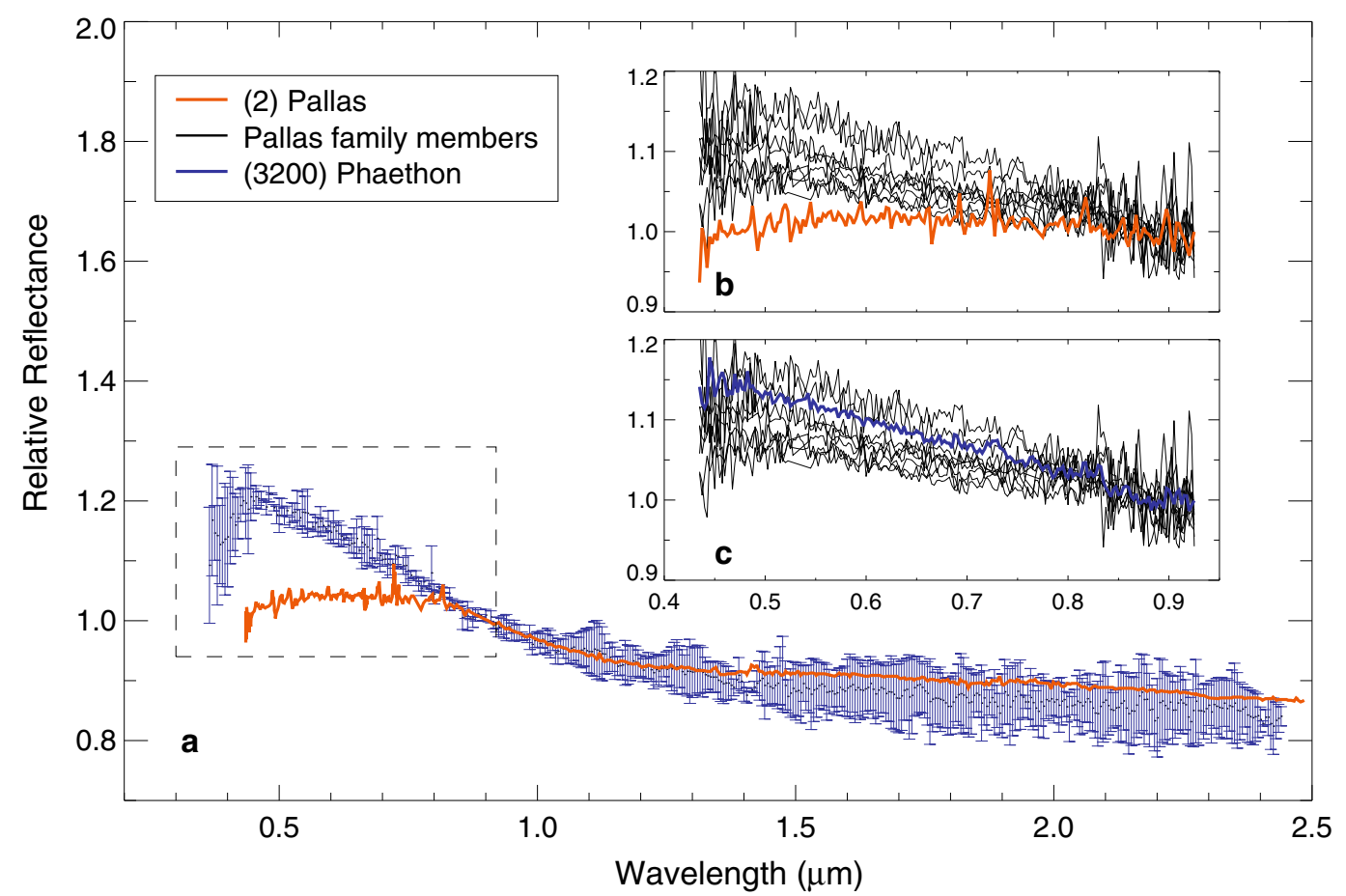

Fig. 2. Spectral comparison between asteroid Phaethon, Pallas and Pallas family members. a) The visible and near-infrared reflectance spectrum of asteroid Phaethon is shown in blue. This composite spectrum results from averaging the spectra taken from two different databases (Binzel et al. 2008; Licandro et al. 2007), after interpolating with a step of $0.005 \mu \mathrm{m}$. Error bars correspond to 1- $\sigma$ variations. The visible and near-infrared spectrum of Pallas is shown in red, and has been taken from the SMASS database. Both reflectance spectra have been normalized to unity at $0.9 \mu \mathrm{m}$. Their similar behaviour in the near-infrared beyond $1 \mu \mathrm{m}$, suggests a similar composition. The main differences are seen in the visible region $(0.4-0.9 \mu \mathrm{m})$, highlighted by a dashed-line box. b) Visible spectrum of Pallas (red line) compared with the available visible spectra of Pallas family members $(6+3$ objects, see Table 1$)$. All spectra have been taken from the SMASS database and normalized to unity at $0.9 \mu$ m. $\mathbf{c})$ Spectral comparison of the Pallas family members with the mean visible spectrum of Phaethon (blue line). It can be clearly seen that Phaethon has a spectrum different from that of Pallas, but essentially identical to its family members'.

have been trapped in resonance, presumably had their orbital eccentricities increased to values that would render them Mars- or even Earth-crossers.

Before discussing in more detail the dynamical connection between Phaethon and Pallas, we establish a spectroscopic one. As can be seen in Fig. 2a, the spectra of Pallas and Phaethon are quite similar beyond $1 \mu \mathrm{m}$, showing a smooth decay in reflectance with a negative slope, suggesting a similar surface composition. In fact, the only significant differences between the two spectra lie in the visible band, between 0.5 and $0.9 \mu \mathrm{m}$, indicated by a dashed-line box. For this reason we decided to compare the visible spectrum of Phaethon not only with that of Pallas, but also with those of the Pallas family members.

In the visible, Phaethon's spectrum is strikingly similar to those of the Pallas family members (Fig. 2c), while it is markedly different from that of Pallas itself (Fig. 2b). Differences in size can explain these spectral variations. While Pallas has a diameter of $\sim 550 \mathrm{~km}$, its family members have diameters that range from 5 to $26 \mathrm{~km}$. Variations in the reflectance spectra with respect to grain size have been studied before for carbonaceous chondrite meteorites (Johson \& Fanale 1973; Sheppard et al. 2008). In particular Johson \& Fanale studied the reflectance spectra of separates of different sizes of CV3 meteorite Grosnaja, finding that the spectra got bluer (negative spectral slope) and darker with coarser grain size. Hence, it is possible that smaller asteroids are covered with coarser grains. This is also consistent with recent results, showing that smaller asteroids present higher thermal intertia (Delbo et al. 2007). Large objects (e.g. Pallas), covered by dusty regolith, tend to have small thermal inertia because of their fine-powdered surface. Smaller objects could have lost their regolith during the collisional event that created them (in the case of collisional families), not being able to develop or retain a new regolith, thus preserving preferentially larger grains on their surfaces.

\section{Dynamical link between Pallas and Phaethon: long-term evolution of Pallas fragments in the NEA space}

We now show how a dynamical link between Pallas and Phaethon can be established. To show that Pallas fragments can become Mars-crossers and subsequently develop Phaethon-like orbits, we integrated the orbits of fictitious Pallas fragments, initially injected in the 8:3 and 5:2 MMRs that border the Pallas family $^{2}$ (notice that the 8:3 MMR had not been considered as a potential NEA source in the NEO model of Bottke et al. 2000, 2002). First, we generated a population of fragments, their initial conditions being selected using the equations of Gauss from Zappalà et al. (1995) and assuming isotropic ejection from Pallas with $V=300 \mathrm{~m} \mathrm{~s}^{-1}$. We then selected only those particles that were injected in the two resonances, as verified by a short-term numerical integration of their orbits. These resonant particles were then cloned, in order to generate a population of $\sim 1000$ resonant particles. The orbits of the resonant particles were then integrated for a time interval of $100 \mathrm{My}$. During this integration, $\sim 95 \%$ of the particles ended their life by falling on the Sun, while the rest $(\sim 5 \%)$ encountered Jupiter and were swiftly

\footnotetext{
${ }^{2}$ We already demonstrated in Fig. 1b that Pallas family members of the size of Phaethon should have been trapped into these resonances.
} 

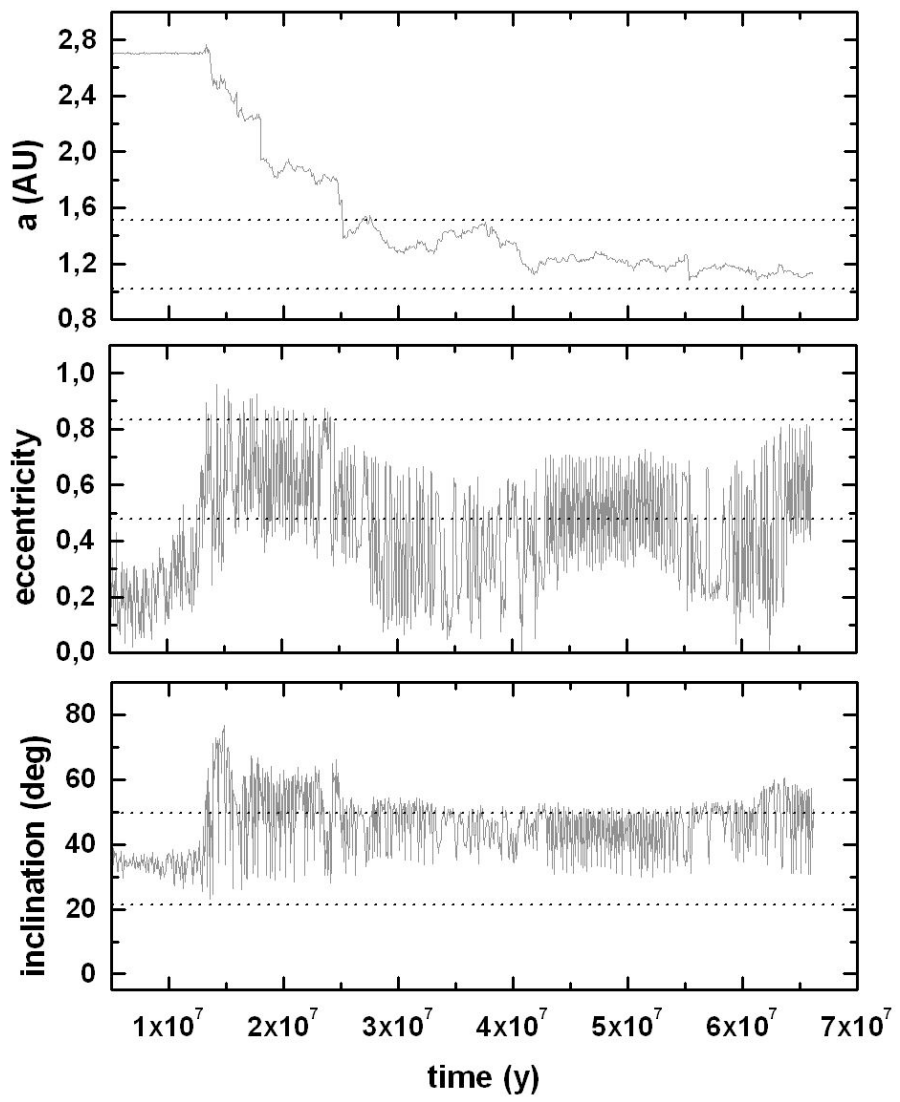

Fig. 3. Dynamical evolution of a fictitious Pallas fragment towards a Phaethon-like orbit. The time evolution of its semi-major axis (top), eccentricity (middle) and inclination (bottom) are shown. The particle was initially injected in the 8:3 MMR with Jupiter. The horizontal dotted lines superimposed on each graph correspond to $\sim 1 \sigma$ deviation from the mean value of the respective orbital parameter of (3200) Phaethon, as computed by integrating the orbital evolution of 21 "clones" of Phaethon for $100 \mathrm{My}$. As shown in these plots, the resonance pumps the eccentricity of this asteroid to higher values, until it becomes an Earth-crosser. Repeated encounters with the Earth extract it from resonance (at $\sim 14 \mathrm{My}$ ) and bring it closer to the terrestrial planets. The particle enters the NEA space $(a<2 \mathrm{AU})$ at $\sim 18 \mathrm{My}$ and spends $\sim 40 \mathrm{My}$ on a high-inclination, Phaethon-like orbit, before it falls on the Sun. Out of $\sim 1000$ particles that we placed initially in the 8:3 MMR, 21 were found to have similar evolutions to the one shown here.

ejected from the system. Of all these objects, 21 (i.e. 2\%) spent several My on Mars- and Earth-crossing orbits with $a<2 \mathrm{AU}$, before ending their life. According to our simulations, Pallas fragments, injected in the 8:3 MMR have a $\sim 3 \times$ higher probability of reaching $a<2$ AU than particles starting from the 5:2 MMR. Figure 3 shows the orbital evolution of a fictitious Pallas fragment starting from the 8:3 MMR. The particle has an eccentricity of $\sim 0.2$ at $t=0$, since it is a Pallas fragment. When it becomes decoupled from the resonance and goes towards the NEA space (around $t=14 \mathrm{My}$ ), its eccentricity starts oscillating between 0 and 0.9 , and when it reaches the NEA region (the last few Myrs), having $a \sim 1.2$ AU and high inclination Phaethonlike orbit, its eccentrity has oscillations that cover almost the same band as Phaethon's statistical sample (i.e. between 0.40 and 0.82 ). So the fictitious Pallas fragment becomes a NEA and develops an orbit quite similar to that of Phaethon.

These results show that there exists a dynamical pathway, through which fragments of Pallas can evolve into Phaethonlike orbits. Thus, Phaethon can be directly linked to the Pallas family, not only spectroscopically but also dynamically. Note that, during this evolution towards the NEA space, the mean inclination changes only by a few degrees. Thus, asteroids escaping from the region of Pallas, which itself follows a highinclination orbit (proper $i \sim 33^{\circ}$ ), would preferentially populate the high-inclination parts of the NEA space, where Phaethon is located.

The mean time interval that an average Pallas fugitive spends within different parts of the $(a, e)$ and $(q, i)$ space (for $a<2$ AU) is shown in Figs. $4 a$ and $b$, where both planes have been divided into $20 \times 20$ cells. As shown in this figure, these Pallas fugitives spend a small, but non-negligible, fraction of their lifetime on Phaethon-like orbits; this is indicated by the proximity of Phaethon to the dark grey region (i.e. cells visited for at least $10^{5.5} \mathrm{yrs}$ ). The orbital space visited by Phaethon is indicated by its mean location, along with 1- $\sigma$ error bars, calculated after an integration of 21 "clones" of Phaethon. A similar computation was done for Rudra (100 Rudra “clones"), a Mars-crossing object whose spectrum is quite close to that of Phaethon and the Pallas family members (see next section) and whose orbit also intersects the region of evolved Pallas fragments. The clones were chosen by adding small, random deviations (of size $10^{-4}$ in $a$ and $e$ and 0.1 in the angles) in the initial values of the orbital elements of Phaethon (resp. Rudra). The orbits of these 21 Phaethon (resp. 100 Rudra) clones were integrated for a time span of 100 My (resp. 150 My) or until they collided with the Sun or one of the planets. The region of orbital elements space covered by these objects, during their evolution, is depicted by computing the mean value and standard deviation in each element $(a, e, i$ and $q$ ), over the entire time interval and over all clones.

Given these results one might expect to find many more B-type NEAs in the black and dark-gray regions of Figs. 4a, b, if the population of B-type NEAs were kept in steady state by a continuous flux of Pallas family members, escaping from their source region. This is not observed. However, Phaethon and Rudra reside on orbits that are some of the longest-lived among those reachable from the Pallas family (see Figs. 4c, d). Indeed, the median dynamical lifetimes of Phaethon and Rudra are $26 \mathrm{My}$ and $106 \mathrm{My}$ respectively, i.e. much longer than the typical NEA dynamical lifetime (a few My). This suggests that the injection of Pallas family members in the 8:3 resonance was higher in the past (presumably during or soon after the familyforming event). In fact, in this case we would expect that the B-type NEAs that still survive today should be in the longestlived orbits, in agreement with the observed orbital positions of Phaethon and Rudra (Figs. 4c, d).

\section{Spectral comparison with other B-types}

For a unique connection between Pallas and Phaethon to be established, one would have to exclude, if possible, all other candidates, or at least show that they are far less likely to be Phaethon's parent bodies than Pallas. The first requirement to be met is the spectral match, both in the visible and the near-infrared. A good match between the complete spectrum of two objects is a strong indication of a similar composition. Unfortunately, there is a lack of near-infrared spectra of B-type asteroids, neither for the Pallas family members nor for other plausible parent bodies, so we restrict our comparison to the visible region. In this sense, data is being currently collected by the authors in the near-infrared of B-type asteroids of the main belt in order to improve the compositional comparison. 

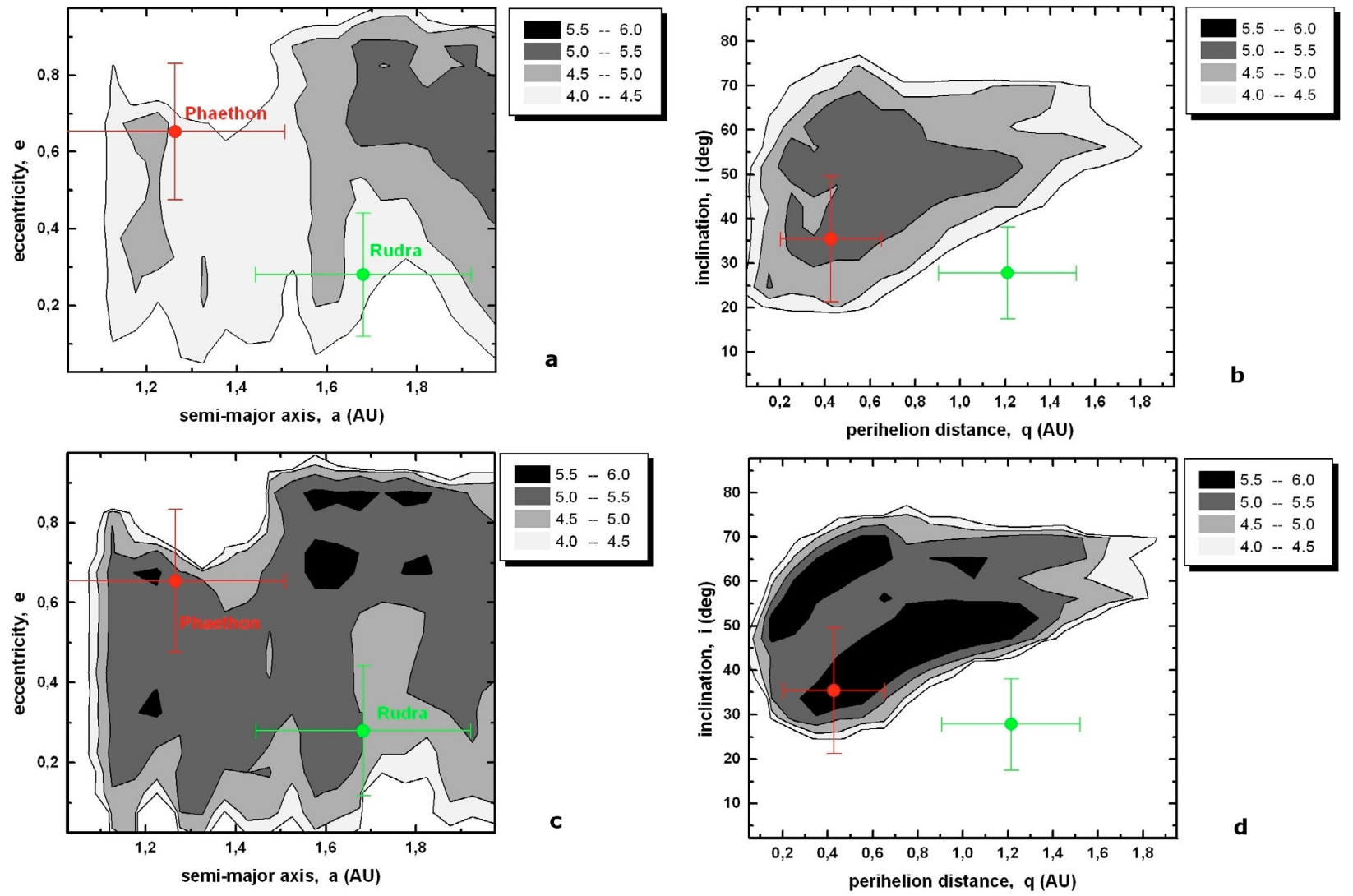

Fig. 4. Long-term orbital evolution in the NEA space. In the top panels $(\mathbf{a})$ and $\mathbf{b}))$ the $(a, e)$ and the $(q, i)$ projections are shown, where $q=a(1-e)$ is the perihelion distance. The quantity plotted in grey-scale is the logarithm of the mean time (in years) that our Pallas-fugitives spend in different regions of the NEA space. Thus, black regions correspond to residence times TR $>10^{5.5} \mathrm{yrs}$, while white regions correspond to TR $<10^{4} \mathrm{yrs}$. Bottom panels (c) and d)) are the same but only particles spending more than $30 \mathrm{My}$ as NEAs are taken into account. The fact that the present positions of Phaethon and Rudra are in better agreement with the residence time distributions of panels c,d than a,b, suggests that the distribution of B-types in the NEA space is not kept in steady state by a continuous flux of Pallas fragments. The mean values of the respective orbital parameters of Phaethon (resp. Rudra) are indicated by a red (resp. green) solid circle. The red (resp. green) error-bars correspond to $\sim 1 \sigma$ deviations from these mean values. As shown here, Phaethon occupies a region of the NEA space that is relatively frequently visited by Pallas fugitives, in both projections. On the other hand, Rudra falls in a less frequently visited portion of the $(a, e)$ space and is more than 1- $\sigma$ away from the long-TR regions of the $(q, i)$ space. This is consistent with the fact that Rudra is an extremely long-lived NEA; its median dynamical lifetime is 106 My.

In order to do so, we have searched all available spectroscopic databases for other objects with visible spectra similar to that of Phaethon, but outside the Pallas family. Spectra for a total of 105 bodies, classified as B-types, were compiled. We use a $\chi^{2}$ test to compare the visible spectra of all selected asteroids with the mean spectrum of Phaethon. The mean spectrum of Phaethon has been computed as a weighted mean of three spectra from 2 different databases (Binzel et al. 2008; Licandro et al. 2007). Weights have been chosen to favour spectra with higher $\mathrm{S} / \mathrm{N}$. The resultant mean spectrum is shown in Fig. 2c. Most of the data we are using for this spectral comparison belongs to SMASS database, but there are also spectra from the S3OS2 (Lazzaro et al. 2004) and NEOSS (de Leon et al. 2010, submitted) databases. Therefore, we apply a wavelength interpolation to all the spectra to yield the same resolution as SMASS data: a step of $0.0025 \mu \mathrm{m}$, from 0.435 to $0.925 \mu \mathrm{m}$. The $\chi^{2}$ statistic is defined as

$\chi^{2}=\sum_{i=1}^{n} \frac{\left(y_{i}-Y_{i}\right)^{2}}{Y_{i}}$

where $y_{i}$ is the reflectance of the asteroid to be compared with Phaethon, $Y_{i}$ is the reflectance of Phaethon and $n$ is the number of data points of the spectra. The smaller the value of $\chi^{2}$ is, the more similar the two spectra are. To avoid the data dispersion usually present at the edges of the visible spectra and due to the lower sensitivity of the detectors at their borders, we compute the statistic between 0.5 and $0.9 \mu \mathrm{m}$. Our criteria to select objects that "best match" the spectrum of Phaethon is the following. We take the $\chi^{2}$ values for the six Pallas family members. The mean $\chi^{2}$ value for these 6 asteroids is 0.121 . Since we are looking for asteroids that match Phaethon's spectrum at least as well as the members of the Pallas family, we will consider as good matches asteroids with $\chi^{2}<0.121$. From a total of 105 objects, only 17 main-belt asteroids and 1 small Marscrosser (Rudra) satisfy our criteria. However, to be considered as a plausible parent body of Phaethon, a spectral match is not enough. The candidate parent bodies should fulfill two additional requirements: (i) be associated with an asteroid family (it is obvious that a B-type family as Pallas is far more likely to be the source of Phaethon than an isolated B-type asteroid that does not present any sign of past fragmentation) and (ii) be positioned close to an effective MMR, which could provide the necessary dynamical path for Phaethon-sized fragments to reach the highinclination part of the NEA space. Thus, unless some of the 17 main-belt bodies we have selected outside the Pallas family satisfy these criteria, they would constitute far less likely sources of Phaethon. Six of these 17 main-belt asteroids belong to the 
collisional family of (24) Themis. This B-type asteroid is the largest object ( $\sim 198 \mathrm{~km}$ in diameter) in its homonymous family, and could constitute a potential source for Phaethon. According to the above discussion, this would be our second best option. However, the infrared spectra of Themis and some of its family members (Clark et al. 2009) are quite different from that of Phaethon (and Pallas). Hence, Themis and its family members, probably have a different composition from Phaethon's. Moreover, this family is located near the 2:1 MMR with Jupiter (at $a \sim 3.2 \mathrm{AU}$ ), which is very unlikely to transport bodies to the near-Earth space (Bottke et al. 2000, 2002), as the probability to produce a low- $a$ NEA decays sharply with increasing semimajor axis of the source. Having low-inclination orbits $\left(\sim 1^{\circ}\right)$, hypothetical Themis fragments, injected in the $2: 1$ resonance, would develop very eccentric orbits, most likely encountering Jupiter and escaping from the Solar System, before they have a chance to become Mars-crossers.

Two more objects were found near the 2:1 MMR with Jupiter, and belong to two high-inclination collisional families: asteroid (1101) Clematis, which belongs to the (702) Alauda family, and asteroid (1901) Moravia, which is the largest member of the Moravia family (Gil-Hutton 2006). Five of the members of the Alauda family have taxonomic classification, with three of them being B-types. In the case of the Moravia family, only two objects have taxonomic classification, and both are B-types. Nevertheless, due to the proximity of these two asteroids to the 2:1 MMR, they are far less likely NEA sources than Pallas. Moreover, we did not find other asteroids, belonging to those families that match Phaethon's spectra. The next six bodies to consider are listed here in increasing order of similarity:

(282) Clorinde, $D=39.03 \mathrm{~km}$

(2809) Vernadskij, $D=6.54 \mathrm{~km}$

(2816) Pien, $D=21.91 \mathrm{~km}$

(4997) Ksana, $D=18.00 \mathrm{~km}$

(142) Polana, $D=55.29 \mathrm{~km}$

(4484) Sif, $D=21.32 \mathrm{~km}$.

Asteroids (282), (2816) and (4997) are isolated B-types, do not have any other dynamically related and smaller asteroids in their surroundings and do not belong to any collisional family. From a dynamical point of view, (4484) Sif is located in a very dispersed (in semi-major axis), high-inclination band of asteroids between the 3:1 ( 2.5 AU) and the 8:3 ( 2.7 AU) MMRs with Jupiter. Thus, dynamically, it appears to be a good candidate, assuming it could inject Phaethon-sized fragments in either of the two resonances. However, although the HCM can identify a family structure around Sif, the values of $V_{\mathrm{c}}$ needed for this $\left(180<V_{\mathrm{c}}<280 \mathrm{~m} \mathrm{~s}^{-1}\right)$ are too large for an asteroid of this small size (only $\sim 21 \mathrm{~km}$ ). Moreover, the largest bodies in this proper elements region, which are linked to the "family" for $V_{\mathrm{c}}>260 \mathrm{~m} \mathrm{~s}^{-1}$, are the S-type asteroid (582) Olympia and the $\mathrm{X} /$ Xc-type (1263) Varsavia. Asteroid (2809) is a good spectral match to Phaethon, but is too small to be considered as a parent body $(D=6.54 \mathrm{~km})$. This asteroid appears to be a member of the Nysa-Polana family, which is not taxonomically homogeneous (Mothé-Diniz et al. 2005): from the 14 asteroids with spectral classification, only 3 are B-types, including (2809). The other two B-types are (3074) Popov and (2973) Paola, which were also included in the spectral comparison and are not in our list of "best matches". So both (2809) and (142) can be excluded as possible sources of Phaethon.
The remaining 4 candidates are dynamically classified as Mars-crossers, since their orbits currently have eccentricities $\sim 0.4$ and perihelion distances $\sim 1.7$ AU: these are (3581) Alvarez, (5690) 1992 UB, (6500) Kodaira and (2629) Rudra. Rudra is a high-inclination $\left(30^{\circ}\right)$ true Mars-crosser. Its orbit intersects the ecliptic at a minimum nodal distance of $\sim 1.35 \mathrm{AU}$. However, this is not true for the other three bodies. Integrating their orbits we found that their perihelion distance becomes $q=1.7 \mathrm{AU}$ only when the argument of perihelion is $\sim 90^{\circ}$. Since their mean inclination is $\sim 33^{\circ}$, this means that they only reach $q=1.7 \mathrm{AU}$ when they are well above or below the ecliptic; their nodal distance is always larger than 1.9 AU, so they never cross the orbit of Mars. More importantly, the secular oscillations of their orbital elements are nearly identical to those found for Pallas; they are just on different phases of very similar evolution cycles. We estimated their proper elements, by timeaveraging their osculating values (see Table 1) over a period of $1 \mathrm{My}$ and found that all three asteroids lie within the borders of the Pallas family. Thus, these three objects are most likely dynamically misclassified members of the Pallas family. This ambiguity comes from the fact that their orbital eccentricities are currently larger $(e \sim 0.4)$ than that of Pallas $(e \sim 0.23)$. This raises the total number of Pallas members that match Phaethon's visible spectrum to nine. Therefore, the last asteroid in our list of possible candidates is (2629) Rudra, a small ( $4.5 \mathrm{~km}$ diameter) Mars-crosser that, therefore, cannot be Phaethon's parent body, but it is likely (see Figs. 4c, d) an object escaped from the Pallas family as well.

The above results show that Phaethon can be directly linked to the Pallas family not only spectroscopically but also dynamically. Since Pallas is the only large B-type asteroid that can be linked both spectroscopically and dynamically with Phaethon, we belive that our results strongly support that Phaethon most likely originated from Pallas. Hence, the Geminids are also very likely pieces of the asteroid Pallas. Furthermore, the Pallas collisional family may constitute an important and previously unidentified source of primitive-type material that can reach the neighborhood of the Earth.

\section{Conclusions}

We have compared reflectance spectra of asteroids Phaethon, Pallas, and all the available spectra of Pallas family members and other main belt objects classified as B-types. Several important conclusions can be extracted from the obtained results.

1. Pallas is the most likely parent body of Phaethon.

2. We found that visible spectrum of Pallas is significantly different from that of its 9 family members, while the one of Phaethon matches strikingly well. We propose differences in asteroid sizes as the most likely explanation for these differences we have found. While Pallas is a large asteroid $(D \sim 550 \mathrm{~km})$, the 9 family members and also Phaethon have diameters of the order of tens of kilometers. Smaller asteroids are probably covered by a coarser regolith, and so their surfaces get bluer and darker. This is consistent with the findings of Delbo et al. (2007), where smaller asteroids have higher thermal inertia, which is indicative of a coarser regolith.

3. Our numerical simulations show the existence of a robust dynamical pathway, connecting the orbital neighborhood of Pallas with that of Phaethon. In this respect, the Pallas family 
may constitute a source of primitive NEAs. Spectral comparison with other main belt asteroids classfied as B-type objects has confirmed the uniqueness of Pallas as the most likely parent body of Phaethon and, consequently, of the Geminids meteor shower.

Acknowledgements. J.deL. and J.L. gratefully acknowledge support from the Spanish "Ministerio de Ciencia e Innovación" projects AYA2005-07808-C03-02 and AYA2008-06202-C03-02. H.C. acknowledges support from NASA's Planetary Astronomy program. H.C. was a visiting Fullbright Scholar at the "Instituto de Astrofísica de Canarias" in Tenerife, Spain. H.C. was a visiting astronomer at the Observatoire de la Côte d'Azur, Nice, France. Part of the data utilized in this publication were obtained and made available by the MITUH-IRTF Joint Campaign for NEO Reconnaissance. The IRTF is operated by the University of Hawaii under Cooperative Agreement No. NCC 5-538 with the National Aeronautics and Space Administration, Office of Space Science, Planetary Astronomy Program. The MIT component of this work is supported by the National Science Foundation under Grant No. 0506716.

\section{References}

Asphaug, E. 1997, Meteor. Planet. Sci., 32, 965

Binzel, R. P., Harris, A., Bus, S. J., \& Burbine, T. 2001, Icarus, 151, 139

Binzel, R. P., Rivkin, A. S., Stuart, J. S., et al. 2004, Icarus, 170, 259

Binzel, R. P., et al. 2008, The MIT-UH-IRTF Joint Campaign for NEO Spectral

Reconnaissance, http://smass.mit.edu/minus.html

Bottke, W. F., Jedicke, R., Morbidelli, A., et al. 2000, Science, 288, 2190

Bottke, W. F., Morbidelli, A., Jedicke, R., et al. 2002, Icarus, 156, 399
Bus, S. J., \& Binzel, R. 2002a, Icarus, 158, 106 Bus, S. J., \& Binzel, R. 2002b, Icarus, 158, 146 Chapman, C. R., \& Salisbury, J. W. 1973, Icarus 19, 507

Clark, B. E., Ziffer, J., Nesvorny, D., et al. 2009, Icarus, submitted Delbo, M., Harris, A. W., Binzel, R. P., et al. 2003, Icarus, 166, 116 Delbo, M., dell'Oro, A., Harris, A. W., et al. 2007, Icarus, 190, 236 de León, J., Licandro, J., Serra-Ricart, M., et al. 2010, A\&A, accepted DeMeo, F. E., Binzel, R. P., Slivan, S. M., \& Schelte, J. B. 2009, Icarus, 202, 160

Emery, J. P., Lim, L. F., Marchis, F., \& Cruikshank, D. P. 2008, LPI Contributions, 1405, 8345

Fowler, J. W., \& Chillemi, J. R. 1992, The IRAS Minor Planet Survey, Technical report

Gil-Hutton, R. 2006, Icarus, 183, 93

Harris, A. W. 1998, Icarus, 131, 291

Hsieh, H. H., \& Jewitt, D. 2006, Science, 312, 561

Johnson, T. V, \& Fanale, F. 1973, JGR, 78, 8507

Johnson, T. V., \& Matson, D. L. 1975, ApJ, 197, 527

Lazzaro, D., Angeli, C. A., Carvano, J. M., et al. 2004, Icarus, 172, 179

Lebofsky, L. A. 1980, AJ, 85, 573

Lemaitre, A., \& Morbidelli, A. 1994, Cel. Mech. Dyn. Astron., 60, 29

Licandro, J., Campins, H., Mothé-Diniz, T., et al. 2007, A\&A, 461, 751

Luu, J., \& Jewitt, D. 1990, AJ, 99, 1985

Mothé-Diniz, T., Roig, F., \& Carvano, J. M. 2005, Icarus, 174, 54

Rivkin, A. S., Howell, E. S., Vilas, F., et al. 2002, in Asteroids III, ed. W. F. Bottke Jr., A. Cellino, P. Paolicchi, \& R. P. Binzel (Tucson: University of Arizona Press), 235

Schmidt, B. E., Thomas, P. C., Bauer, J. M., et al. 2009, Science, 326, 275

Shepard, M., Clark, B. E., Nolan, M. C., et al. 2008, Icarus, 193, 20

Tedesco, E. F. 1992, The IRAS Minor Planet Survey, Tech. Rep. PLTR-92-2049, Phillips Laboratory, Hanscom Air Force Base, MA

Tholen, D. J. 1984, Ph.D. Thesis, AA (Tucson: Arizona University)

Whipple, F. L., IAU Circ., 3881

Zappala, V., Bendjoya, Ph., Cellino, A., et al. 1995, Icarus, 116, 291 